\title{
Eccrine Angiomatous Hamartoma in an Adolescent
}

\author{
Pimpa Tantanasrigul Tanawatt Kootiratrakarn Vesarat Wessagowit \\ Pinnaree Kattipathanapong Poonnawis Sudtikoonaseth \\ Institute of Dermatology, Ministry of Public Health, Bangkok, Thailand
}

\section{Key Words}

Eccrine angiomatous hamartoma $\cdot$ D2-40 $\cdot$ CD31 $\cdot$ Anti-Ulex europaeus- 1

\begin{abstract}
Eccrine angiomatous hamartoma is a rare hamartoma that usually affects childhood and adolescence. In this report we describe a typical onset and clinical presentation of eccrine angiomatous hamartoma. Histopathological analysis and immunohistochemical staining of the sections were done to confirm the diagnosis.

(C) 2015 The Author(s)

Published by S. Karger AG, Basel
\end{abstract}

\section{Case Report}

An 18-year-old Thai woman came to our outpatient clinic with a lump on her right knee. The mass had first been noticed 1 year before and had grown slowly without regression. She complained of tenderness on palpation. A history of local preceding trauma had been noted. The patient had gone to another hospital prior to this visit and a needle aspiration had been performed without and content being obtained from the procedure. Her medical history was unremarkable. Physical examination revealed a solitary well-defined bluish mass, approximately $5.0 \times 3.0 \times 0.5 \mathrm{~cm}$ in size, with firm consistency located on the right knee (fig. 1). A skin biopsy was done in which the hematoxylin and eosin-stained section displayed increasing components of blood vessels and mature eccrine glands in the mid to deep dermis (fig. 2). Adipose tissue was generally observed. The epidermis was unremarkable. The section showed positive staining with D2-40, CD31 and anti-Ulex europaeus-1, whereas the staining result for GLUT-1 was negative. Magnetic resonance imaging was performed to identify the outline and depth of the lesion. The magnetic resonance imaging result showed an enhancing cutaneous nodule with surrounding subcutaneous tissue edema. Since the 
Tantanasrigul et al.: Eccrine Angiomatous Hamartoma in an Adolescent

patient complained of an increasing intensity of pain when she came back for follow-up, she was referred to a surgeon for mass excision.

\section{Discussion}

Eccrine angiomatous hamartoma $(\mathrm{EAH})$ is a rare benign tumor of hamartomatous origin. Its etiology is yet to be elucidated. EAH may occur as a result of an abnormal interaction between the differentiated epithelium and the mesenchyme, leading to atypical proliferation of eccrine and vascular elements [1]. EAH was once described as sudoriparous angioma [2]. Nevertheless, sudoriparous angioma was later discussed by some authors as another distinct entity since it showed dilatation instead of proliferation of the eccrine structures [3].

EAH is primarily a disease of the young, with onset usually during childhood and adolescence. In recent years, however, there has been a rising number of cases reported as adulthood EAH in the literature [4]. The disease shows no gender or racial preponderance. EAH commonly presents as a single nodule or papule; multiple lesions are uncommon. The color of the tumor varies individually from flesh-colored, yellow-brown to bluish. The predilection sites are the distal extremities, particularly the palms and soles. Some of the lesions may be exclusively accompanied by hyperhidrosis or hypertrichosis. Both features have therefore been valued as clues for diagnosis. In general EAH is asymptomatic, but associated pain may occur.

The definite diagnosis of EAH requires microscopic evaluation. A histopathological hallmark is the proliferation of eccrine and vascular structures, capillaries in particular. The level of pathology is often located in the mid to deep dermis; the epidermis is usually unremarkable. Nerves may sometimes be observed from the section and its presentation is associated with pain symptoms. The criteria for the diagnosis of EAH were proposed by Pelle et al. [5] as follow: (1) hyperplasia of normal or dilated eccrine glands, (2) close association of the eccrine structures with capillary angiomatous foci, and (3) variable presence of pilar, lipomatous, mucinous and/or lymphatic structures. Smith et al. [6] also designated three histological variants of EAH; follicular, lipomatous and mucinous. Some anecdotal cases of EAH with verrucous epidermal hyperplasia as well as features overlapping with hemangioma have been reported [7].

Immunohistochemical staining in EAH delineates normal eccrine structures. The secretory portions of the eccrine gland show positivity for S100, CAM 2.5, anti-EMA and anti-CEA, whereas the ductal portions can be labelled by anti-CEA, cytokeratin and anti-EMA [8]. Factor VIII-related antigen and anti-Ulex europaeus-1 are useful for identifying the vascular components [9]. Lymphatic vessels can be recognized with D2-40 staining. The main differential diagnoses for EAH are other vascular malformation, blue rubber bleb nevus, tufted angioma, glomus tumor and smooth muscle hamartoma.

EAH runs a benign course without spontaneous regression or malignant transformation. The lesion may enlarge in proportion with the patient's growth. Recurrence may occur. The definite therapeutic management is surgical excision [1]. Laser depilation may be benefit in the presence of hypertrichosis. Botulinum toxin injection has been reported to show satisfying result in lesions accompanied by hyperhidrosis [10]. 


\section{Case Reports in Dermatology}

Tantanasrigul et al.: Eccrine Angiomatous Hamartoma in an Adolescent

\section{Acknowledgement}

We would like to express our deep gratitude to Dr. Poonnawis Sudtikoonaseth, MD, who assisted with the histopathological review.

\section{Statement of Ethics}

The authors have no ethical conflicts to disclose.

\section{Disclosure Statement}

The authors declare no conflict of interest.

\section{References}

1 Martinelli PT, Tschen JA: Eccrine angiomatous hamartoma: a case report and review of the literature. Cutis 2003;71:449-455

2 Domonkos AN, Suarez LS: Sudoriparous angioma. Arch Dermatol 1967;96:552-553.

-3 Nakatsui TC, Schloss E, Krol A, Lin AN: Eccrine angiomatous hamartoma: report of a case and literature review. J Am Acad Dermatol 1999;41:109-111.

4 Shin J, Jang YH, Kim SC, Kim YC: Eccrine angiomatous hamartoma: a review of ten cases. Ann Dermatol 2013;25:208-212.

5 Pelle MT, Pride HB, Tyler WB: Eccrine angiomatous hamartoma. J Am Acad Dermatol 2002;47:429-435.

6 Smith VC, Montesinos E, Revert A: Eccrine angiomatous hamartoma: report of three patients. Pediatr Dermatol 1996;13:139-142.

7 Cheong SH, Lim JY, Kim SY, Choi YW, Choi HY, Myung KB: A case of eccrine angiomatous hamartoma associated with verrucous hemangioma. Ann Dermatol 2009;21:304-307.

-8 Cebreiro C, Sanchez-Aguilar D, Gomez Centeno P, Fernandez-Redondo V, Toribio J: Eccrine angiomatous hamartoma: report of seven cases. Clin Exp Dermatol 1998;23:267-270.

-9 Garcia-Arpa M, Rodriguez-Vazquez M, Cortina-de la Calle P, Romero-Aguilera G, Lopez-Perez R: Multiple and familial eccrine angiomatous hamartoma. Acta Derm Venereol 2005;85:355-357.

$\checkmark 10$ Barco D, Baselga E, Alegre M, Curell R, Alomar A: Successful treatment of eccrine angiomatous hamartoma with botulinum toxin. Arch Dermatol 2009;145:241-243. 


\section{Case Reports in Dermatology}

Tantanasrigul et al.: Eccrine Angiomatous Hamartoma in an Adolescent

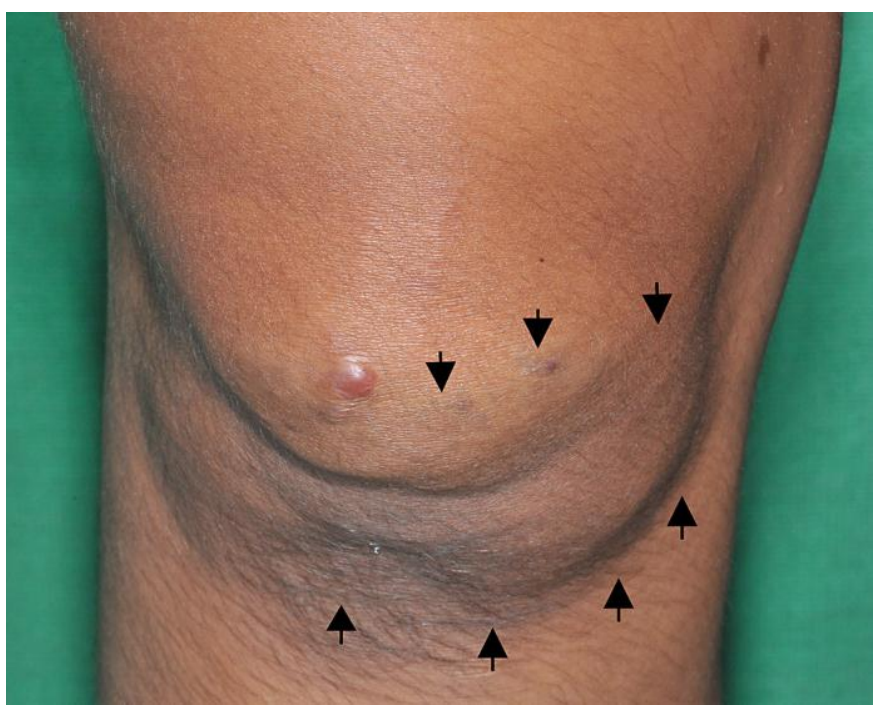

Fig. 1. A well-defined bluish mass on the right knee (arrows).

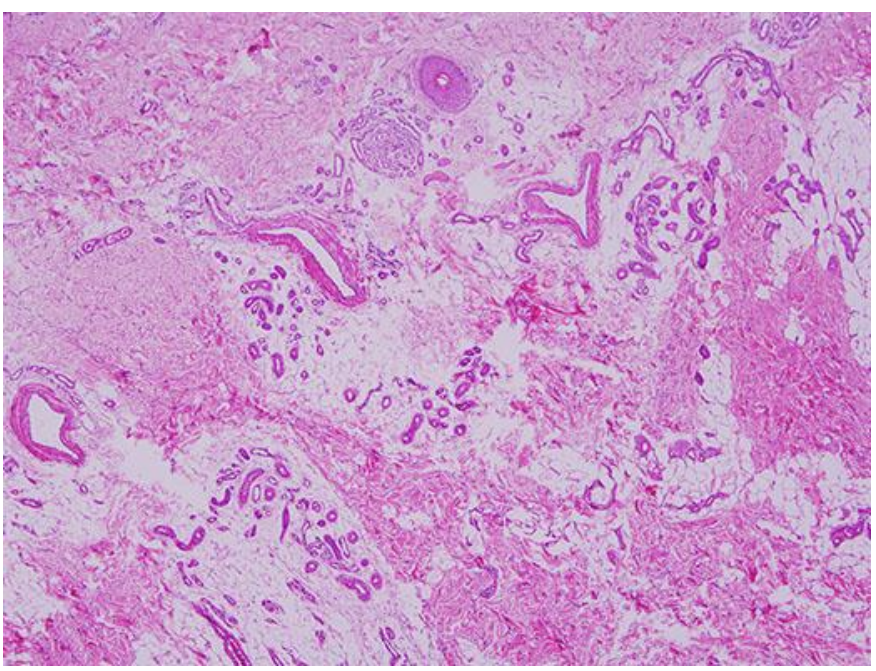

Fig. 2. Histopathological image showing proliferation of both vascular components and eccrine structures in the mid to deep dermis (hematoxylin and eosin, original magnification $\times 4$ ). 